Díaz-García, I., Almerich, G., Suárez-Rodríguez, J. y Orellana, N. (2020). La relación entre las competencias TIC, el uso de las TIC y los enfoques de aprendizaje en alumnado universitario de educación. Revista de Investigación Educativa, 38(2), 549-566.

DOl:http://dx.doi.org/10.6018/rie.409371

\title{
La relación entre las competencias TIC, el uso de las TIC y los enfoques de aprendizaje en alumnado universitario de educación
}

\section{The relationship between ICT competences, ICT use and learning approaches in university students of education}

\author{
Isabel Díaz-García, Gonzalo Almerich Jesús Suárez-Rodríguez y Natividad Orellana \\ Departamento Métodos de Investigación y Diagnóstico en Educación. Facultad de Filosofía y Ciencias de la Educa- \\ ción. Universidad de Valencia. España
}

\begin{abstract}
Resumen
Las tecnologías de la información y de la comunicación (TIC) suponen un cambio en los modos de aprendizaje del alumnado universitario. El propósito de este estudio es conocer la relación entre los enfoques de aprendizaje (superficial y profundo), el uso de las TIC (uso personal, uso académico no escolar y uso académico en el aula) y las competencias TIC (tecnológicas, pedagógicas y éticas) en alumnado universitario de titulaciones de Educación. Se trata de un estudio basado en un diseño correlacional, cuya muestra la constituyen 485 estudiantes de las titulaciones de grado de Pedagogía y de Educación Social de la Universidad de Valencia, seleccionados mediante un muestreo no probabilístico y accidental, recogiendo la información mediante dos cuestionarios. Los resultados señalan la existencia de un vínculo complejo entre las competencias TIC, el uso de las TIC y los enfoques de aprendizaje. Se ha de destacar la relación entre las competencias pedagógicas y las éticas, el uso académico no escolar y el enfoque profundo, así como la relación entre enfoque superficial y uso académico de las TIC en el aula. Por ello, se sugiere la necesidad de formar a los estudiantes en las competencias pedagógicas y éticas de las TIC, ya que si se hiciera se estaría fomentando el aprendizaje profundo.

Palabras clave: tecnologías de la información; estudiantes universitarios; competencia; usos de la tecnología en educación; aprendizaje.

Correspondencia: Isabel Díaz García. Isabel.Diaz-Garcia@uv.es. Facultad de Filosofía y Ciencias de la Educación. Departamento MIDE. Avda. Blasco Ibáñez, 30. 46020 Valencia (Spain)
\end{abstract}




\begin{abstract}
Information and communication technologies (ICT) represent a change in the ways of learning of university students. The purpose of this study is to know the relationship between learning approaches (superficial and deep), use of ICT (personal use, non-school academic use and academic use in the classroom) and ICT competences (technological, pedagogical and ethical) in university students of Education degrees. This is a study based on a correlational design, whose sample is made up of 485 students of the degrees of Pedagogy and Social Education at the University of Valencia, selected by non-probabilistic and accidental sampling, collecting the information through two questionnaires. The results indicate the existence of a complex link between ICT competences, use of ICT and learning approaches. The relationship between pedagogical and ethical competences, non-school academic use and an in-depth approach, and between a superficial approach and academic use of ICT in the classroom should be highlighted. Therefore, the need to train students in the pedagogical and ethical competences of ICT is stressed in order to encourage deep learning.

Keywords: information technology; college students; competence; technology uses in education; learning.
\end{abstract}

\title{
Introducción
}

En la actual Sociedad del Conocimiento es imprescindible que la ciudadanía adquiera nuevas competencias para poder responder a las demandas que reclaman los cambios producidos por la evolución constante de la tecnología. Estas competencias se convierten en elementos esenciales de cambio en el nuevo paradigma educativo (Martínez-Clares \& González-Lorente, 2019). Consiguientemente, la educación se convierte en un ámbito social pleno de relevancia, pues ha de formar en la adquisición de estas nuevas competencias a las ciudadanas y los ciudadanos que se han de integrar en la sociedad actual y futura, apropiándose el sistema educativo de una trascendencia extraordinaria.

Estas nuevas competencias transversales requeridas constituyen, por una parte, lo que se ha denominado competencias del siglo XXI, que se componen de competencias de alta habilidad y las competencias referidas a las tecnologías de la información y de la comunicación (TIC) (Almerich, Suárez-Rodríguez, Díaz-García \& Orellana, 2020). Por otra, se encuentran las competencias clave, de las que forma parte la competencia digital o competencia TIC (Comisión Europea, 2018).

La universidad también se ve afectada por estos cambios. El alumnado se enfrenta con la necesidad de adquirir nuevas competencias para una sociedad en la cual la tecnología es clave (Comisión Europea, 2017), y que van más allá de las comprensiones conceptuales y los modos disciplinarios de pensar y practicar (McCune \& Entwistle, 2011). Estos cambios han supuesto que el modelo de aprendizaje del alumnado universitario actual sea diferente.

Por un parte, el alumnado universitario se encuentra en un contexto de aprendizaje en el que los recursos tecnológicos forman parte del proceso de enseñanza-aprendizaje. Consecuentemente, las competencias TIC son una competencia clave y transversal que el alumnado ha de adquirir a lo largo de su formación (Comisión Europea, 2018). 
Asimismo, las competencias TIC se erigen en fundamentales para la adquisición de competencias de alta habilidad (Almerich et al., 2020).

Por otra parte, el alumnado es consciente que el empleo de métodos efectivos de pensamiento y cómo aprenden mejora la forma en la que aprenden (Nugent et al., 2019). Además, el aprendizaje profundo y significativo facilita tanto la adquisición de conocimientos y la solución a las tareas de resolución de problemas como su proceso de aprendizaje (Nugent et al., 2019). Igualmente, el aprendizaje está influido por el enfoque de aprendizaje adoptado por el alumnado, afrontándolo desde un enfoque profundo o un enfoque superficial (Monroy \& Hernández Pina, 2014).

La manera en la que el alumnado universitario concibe el aprendizaje conlleva procesos más o menos complejos que determinan un enfoque de aprendizaje (Maquilón Sánchez, Mirete Ruiz, García Sánchez, \& Hernández Pina, 2013). Asimismo, el uso personal y/o académico de las TIC se relaciona con un tipo de enfoque de aprendizaje $\mathrm{u}$ otro, donde el resultado puede ser un aprendizaje profundo o meramente superficial. No obstante, la relación entre el enfoque del aprendizaje del alumnado -profundo y superficial-, las competencias en TIC y el uso de las TIC personal y académico no ha sido explorada suficientemente desde un punto de vista empírico. Por ello, en este estudio se responde a dos preguntas sobre el alumnado universitario del ámbito educativo:

- ¿Cómo se relacionan las competencias TIC, el uso de las TIC y los enfoques de aprendizaje?

- ¿Qué dimensiones de las competencias TIC, el uso de las TIC y los enfoques de aprendizaje son determinantes y contribuyen a la relación?

\section{Las competencias y el uso de las TIC}

El alumnado universitario, para desenvolverse adecuadamente y tener éxito en la sociedad actual, ha de adquirir el dominio de las competencias TIC (Almerich et al., 2020) y usar los recursos tecnológicos de forma adecuada (Díaz-García, Almerich, Cebrián-Cifuentes \& Suárez-Rodríguez, 2019). Por ello, tanto las competencias como el uso de la TIC son fundamentales en su aprendizaje, actual y futuro.

Las competencias TIC, o competencias digitales, son el conjunto de conocimientos y habilidades que posee el alumnado referente al dominio de diferentes recursos tecnológicos y su consideración en sus diversas tareas académicas y/o actividades formativas, desde un punto de vista ético, seguro y responsable. A lo largo de estos años, diversas organizaciones y autores han formulado distintos marcos competenciales en TIC tanto para el alumnado en general como para el alumnado universitario (Arras, Torres, \& García-Valcarcel, 2011; ISTE, 2016; REBIUN, 2016; Vuorikari, Punie, Carretero Gómez, \& Van den Brande, 2016). Estas diversas propuestas no reflejan cierto acuerdo, lo que dificulta su visión global. No obstante, a partir de Almerich et al. (2020) las competencias TIC del alumnado, universitario y no universitario, se estructuran en tres subconjuntos competenciales:

Las competencias tecnológicas, que hacen referencia a aquellos conocimientos y habilidades que permite al alumnado dominar los recursos tecnológicos. 
- Las competencias pedagógicas, que se refieren a la integración que el alumnado hace de los recursos tecnológicos para la realización de las tareas académicas y/o actividades formativas.

- Las competencias éticas, para referirse tanto al uso legal que se hace de las aplicaciones como respecto al reconocimiento de la autoría, en las tareas académicas y/o formativas por parte de los estudiantes.

Respecto al uso de las TIC por parte del alumnado se refiere a la frecuencia temporal de uso de los distintos recursos tecnológicos para la realización de distintas tareas que adquieren complejidad, ya sea para propósitos personales y/o académicos y que tienen lugar tanto en el aula como fuera de la misma. A partir de Díaz-García et al. (2019), el uso del alumnado se puede enmarcar en tres ámbitos:

- Uso personal, que se refiere a la frecuencia de uso de los recursos tecnológicos que realiza para diversas tareas no académicas fuera del centro educativo.

- Uso académico no escolar, que alude a la frecuencia de uso de los recursos tecnológicos que realiza para diversas tareas académicas fuera del centro educativo.

- Uso académico en el aula, que hace referencia a la frecuencia de uso de los recursos tecnológicos que realiza para diversas tareas académicas dentro del aula.

\section{Los enfoques de aprendizaje}

El alumnado cuando se enfrenta a las tareas académicas en su entorno lo realiza desde la percepción que tiene de la tarea y sus atributos (Gargallo, Garfella \& Pérez, 2006), lo que le conduce a la aplicación de diferentes procesos de aprendizaje (McCune \& Entwistle, 2011). Así, Biggs y Tang (2011) utilizan el término enfoques de aprendizaje para referirse a los modos de aprender una tarea determinada, pero sin describir las características del alumnado.

Desde que Marton y Säljö (1976a,b) acuñaron los términos enfoque del aprendizaje profundo y superficial, a lo largo del tiempo diferentes escuelas han reafirmado estos dos constructos (Soler-Contreras, Cárdenas-Salgado, Hernández-Pina \& MonroyHernández, 2017).

El enfoque de aprendizaje profundo se caracteriza porque el alumnado desea lograr que el aprendizaje tenga significación personal, obteniendo un nivel de comprensión profundo e integrando los principios fundamentales y los hechos, y donde se pretende relacionar ideas y establecer conexiones con el propio conocimiento (Gargallo et al., 2006; Monroy \& Hernández Pina, 2014). El enfoque de aprendizaje superficial se caracteriza porque el alumnado trata de cumplir con los requisitos de la evaluación, con un nivel de comprensión superficial y sin integrar los principios, sin pretender establecer relaciones entre ideas y conexiones con el propio conocimiento (Gargallo et al., 2006; Monroy \& Hernández Pina, 2014).

Pese a esta distinción entre enfoque de aprendizaje profundo y superficial, McCune y Entwistle (2011) señalan que en los enfoques de aprendizaje existen elementos tanto 
de variabilidad como de estabilidad. De esta forma, Biggs y Tang (2011) indican que el alumnado muestra una predisposición o preferencia por un determinado enfoque de aprendizaje, si bien el enfoque que pone en práctica el alumnado para una determinada tarea depende del contexto de la enseñanza.

\section{La relación entre competencias TIC, uso de las TIC y los enfoques de aprendizaje}

La relación entre los tres constructos ha sido estudiada parcialmente en diferentes investigaciones. Sin embargo, no se ha abordado de forma conjunta.

En el caso de la relación entre la competencia TIC y el uso de las TIC, Rohatgi, Scherer y Hatlevik (2016) encontraron una relación explicativa positiva entre el uso de las TIC respecto de las competencias TIC, mediada por la autoeficacia en las TIC básica. En otro estudio, Hatlevik, Ottestad y Throndsen (2015) obtuvieron una relación explicativa negativa por parte del uso de las TIC en la escuela con las competencias TIC.

Referente a la relación entre el uso de las TIC y los enfoques de aprendizaje, Maquilón Sánchez et al. (2013) hallaron en su estudio una vinculación positiva entre la valoración de las TIC por parte del alumnado y el enfoque profundo. Díaz-García et al. (2019) encontró que el uso académico no escolar se vincula con el enfoque profundo, y el superficial con el uso personal.

Finalmente, la relación entre los enfoques de aprendizaje y la competencia TIC no se ha encontrado en la revisión realizada. Se ha de destacar que Richardson, Abraham y Bond (2012) afirman que los enfoques de aprendizaje se relacionan con el rendimiento académico del alumnado, negativo en el caso del enfoque superficial y positivo para el enfoque profundo. Es esperable que la relación entre competencias TIC siga esta vinculación.

\section{Método}

\section{Objetivos}

El objetivo general del estudio es conocer la relación entre los conjuntos competencias TIC -competencias tecnológicas, competencias pedagógicas y competencias éticas-, uso de las TIC - personal, académico no escolar y académico en el aula- y enfoques de aprendizaje -profundo y superficial- en alumnado universitario del ámbito de la Educación. En concreto se tratan dos objetivos específicos:

a) Determinar la relación entre los conjuntos de las competencias TIC, el uso de las TIC y los enfoques de aprendizaje.

b) Determinar las dimensiones de cada uno de los conjuntos anteriores que contribuyen a la relación estudiada.

\section{Población y Muestra}

La población se define como el total de los estudiantes universitarios de titulaciones del ámbito de educación de la Universidad de Valencia. La muestra, seleccionada mediante un muestreo no probabilístico accidental, está constituida por 
485 estudiantes de los grados de Pedagogía (52.8\%) y Educación Social (47.2\%) de la Universidad de Valencia. El $86.8 \%$ son mujeres y el 13.2\% son hombres, siendo la edad media de los participantes de 21.3 años en un rango que va de 18 a 53 años. El 100\% dispone de ordenador y teléfono móvil y el $98.5 \%$ dispone de conexión a Internet. Los estudiantes utilizan siempre o casi siempre el ordenador e Internet, tanto para uso personal como académico.

\section{Instrumento}

En este estudio se han utilizado dos cuestionarios. El primero es el protocolo Innovatic (Suárez-Rodríguez, Almerich, Orellana \& Díaz-García, 2016), que recoge información sobre diversos apartados relativos a las TIC para alumnado universitario. Los factores considerados en este estudio son:

- Puntuación global media de la competencia tecnológica (rango 1-5). En esta dimensión se le pregunta al alumnado sobre el dominio de diferentes recursos tecnológicos, evaluados mediante una escala tipo Likert que consta de 42 ítems y con cinco opciones de respuesta. La fiabilidad de la escala obtenida en este estudio es $\alpha$ de Cronbach= .94.

- Puntuación global media de la competencia pedagógica en TIC (rango 1-5). En esta dimensión se le pregunta al alumnado acerca de cómo llevan a cabo la integración de las TIC en sus tareas académicas y/o acciones formativas, evaluados mediante una escala tipo Likert que consta de 34 ítems y con cinco opciones de respuesta. La fiabilidad de la escala obtenida en este estudio es $\alpha$ de Cronbach $=.90$.

- Puntuación global media de la competencia ética en TIC (rango 1-5). En esta dimensión se le pregunta al alumnado sobre los aspectos éticos, que tienen que ver tanto con el uso legal que se hace de las aplicaciones como sobre el reconocimiento de la autoría, en sus tareas académicas y/o acciones formativas, evaluados mediante una escala tipo Likert que consta de 9 ítems, y con cinco opciones de respuesta. La fiabilidad de la escala obtenida en este estudio es $\alpha$ de Cronbach $=.83$.

- Puntuación global media del uso personal de las TIC (rango 1-5). En esta dimensión se le pregunta al alumnado sobre el uso que realizan de diversos recursos tecnológicos para propósito personal, evaluados mediante una escala tipo Likert que consta de 39 ítems y con cinco opciones de respuesta. La fiabilidad de la escala obtenida en este estudio es $\alpha$ de Cronbach $=.90$.

- Puntuación global media del uso académico no escolar de las TIC (rango 1-5). En esta dimensión se le pregunta al alumnado sobre el uso que realizan de diversos recursos tecnológicos para propósito académico en su domicilio, evaluados mediante una escala tipo Likert que consta de 39 ítems, y con cinco opciones de respuesta. La fiabilidad de la escala obtenida en este estudio es $\alpha$ de Cronbach $=.89$.

- Puntuación global media del uso académico en el aula de las TIC (rango 1-5). En esta dimensión se le pregunta al alumnado sobre el uso que realizan de diversos 
recursos tecnológicos en el aula, evaluados mediante una escala tipo Likert que consta de 39 ítems, y con cinco opciones de respuesta. La fiabilidad de la escala obtenida en este estudio es $\alpha$ de Cronbach $=.93$.

El segundo instrumento es el Cuestionario de Procesos de Estudio en su versión de dos factores (Biggs, Kember \& Leung, 2001), que evalúa los enfoques de aprendizaje. Comprende 20 ítems, divididos en dos escalas, una de enfoque superficial y otra de enfoque profundo, cada una de las cuales está formada por 10 ítems que se subdividen en dos factores, que son las utilizadas en este estudio. El cuestionario adopta el formato de las escalas tipo Likert con cinco opciones de respuestas. En este estudio, la fiabilidad de la escala obtenida del enfoque superficial es $\alpha$ de Cronbach $=.82$, y la de la escala del enfoque profundo es $\alpha$ de Cronbach $=.83$.

\section{Diseño de investigación, procedimiento de recogida y análisis de datos}

El diseño de investigación utilizado es un estudio correlacional, de tipo transversal dado que se ha recogido la información en una única administración.

El procedimiento de recogida de la información se ha llevado a cabo durante los cursos 2016-17 y 2017-2018, mediante cuestionarios en papel. En la investigación se tuvieron en cuenta todas las directrices que requiere el Comité de Ética de la Universidad de Valencia para la realización de este tipo de investigación, explicando al alumnado el objetivo del estudio y la importancia de su respuesta, señalándoseles la confidencialidad de su respuesta y el anonimato de los datos, tanto verbalmente como de forma escrita. Además, su participación fue voluntaria y con su consentimiento, sin ningún tipo de incentivo por su colaboración.

Los análisis de datos, obtenidos mediante el programa SPSS 24.0, son estadísticos descriptivos, correlación de Pearson y Correlación Canónica no lineal.

Los indicadores que se han considerado para el tamaño del efecto son la r2, en el caso de la correlación de Pearson, y la R2, en el caso de la correlación canónica no lineal. Atendiendo a Cohen, Cohen, West y Aiken (2003) los valores de referencia son en ambos casos: $1 \%-8.9 \%$ pequeño; $9 \%-24,9 \%$ mediano; $\geq 25 \%$ grande.

\section{Resultados}

Los resultados se presentan en dos apartados. El primero corresponde a los estadísticos descriptivos de las dimensiones estudiadas de los tres conjuntos: enfoques de aprendizaje, las competencias TIC y el uso de los recursos tecnológicos, junto con las correlaciones entre las dimensiones. En el segundo se presenta la relación entre las competencias TIC, los usos de las TIC y los enfoques de aprendizaje.

\section{Estadísticos descriptivos}

Respecto a los enfoques de aprendizaje -ver tabla 1-, el alumnado presenta mayores puntuaciones en el enfoque profundo que en el enfoque superficial. Consideran a menudo el enfoque profundo en las tareas académicas a realizar, mientras que algunas veces en 
el enfoque superficial. A partir de la desviación estándar, el alumnado presenta una cierta heterogeneidad en las respuestas. Si se considera la asimetría y la curtosis, existe más alumnado por debajo de la media y agrupados ligeramente alrededor de la misma.

En lo que se refiere a las competencias TIC, el valor mayor lo obtienen en las competencias éticas, seguidas de las pedagógicas y las tecnológicas -ver tabla 1-. En cuanto a las competencias tecnológicas, el alumnado presenta un nivel de domino medio de los diversos recursos tecnológicos, con limitaciones en la funcionalidad de los recursos. Respecto a las competencias pedagógicas el nivel es medio, pues el alumnado considera las TIC en sus tareas académicas y/o formativas algunas veces. Por último, en las competencias éticas el nivel encontrado también es medio, dado que tiene en cuenta los aspectos legales y éticos en algunas ocasiones. A partir de la desviación estándar, el alumnado presenta una cierta heterogeneidad en las respuestas en las competencias éticas, no en las tecnológicas y pedagógicas que muestra homogeneidad. Si se considera la asimetría y la curtosis, en el caso de la competencia tecnológica existe más alumnado por debajo de la media y agrupados ligeramente alrededor de la misma. En el caso de las competencias pedagógicas y éticas existe más alumnado por encima de la media y agrupados alrededor de la misma en las competencias pedagógicas y no en las éticas, pues existe un mayor reparto a lo largo de la distribución.

En cuanto a uso de las TIC -ver tabla 1-, el alumnado muestra el valor medio más alto en el uso personal, ligeramente superior al uso académico no escolar, y el menor en el uso académico en el aula. En el uso personal y en el académico no escolar el nivel de uso de las TIC es medio, mientras que en el uso en el aula el nivel es medio-bajo. A partir de la desviación estándar, el alumnado presenta una cierta heterogeneidad en las respuestas en los tres usos considerados. Si se considera la asimetría y la curtosis, en los tres tipos de uso existe más alumnado por encima de la media y agrupados alrededor de la misma, esencialmente en el uso académico no escolar.

Tabla 1

Estadísticos descriptivos

\begin{tabular}{lcccc}
\hline \multicolumn{1}{c}{ Dimensiones } & Media & $\begin{array}{c}\text { Desviación } \\
\text { estándar }\end{array}$ & Asimetría & Curtosis \\
\hline Enfoque superficial & 2.22 & .72 & .76 & .46 \\
Enfoque profundo & 2.83 & .69 & .49 & .22 \\
Competencia tecnológica & 3.02 & .50 & .40 & .24 \\
Competencia pedagógica & 3.55 & .49 & -.19 & .34 \\
Competencia ética & 3.62 & .73 & -.31 & -.31 \\
Uso personal & 2.82 & .50 & .16 & .07 \\
Uso académico no escolar & 2.75 & .55 & .61 & .80 \\
Uso académico en el aula & 2.44 & .62 & .42 & -.06 \\
\hline
\end{tabular}


Referente a las relaciones entre las dimensiones de un mismo conjunto -ver tabla 2, las correlaciones entre los tres componentes de las competencias TIC son positivas. La intensidad de la relación oscila de .198 a .487 , con un tamaño del efecto pequeño (competencias tecnológicas-competencias éticas) y mediano (competencias pedagógicas con las tecnológicas y las éticas). En cuanto al uso de las TIC, las correlaciones entre las tres dimensiones son positivas, con una intensidad de la relación que oscila de .653 a .791, y con un tamaño del efecto grande en las tres vinculaciones. Finalmente, el enfoque profundo y el enfoque superficial muestran una correlación negativa, con una intensidad de .198, que supone un tamaño del efecto pequeño.

Respecto a las correlaciones entre las dimensiones de las competencias TIC y las del uso de las TIC, en todos los casos son positivas. Consiguientemente, a mayor competencia TIC mayor uso de las TIC, y viceversa. Las competencias tecnológicas muestran mayor intensidad de la relación con los tres usos de las TIC, y en menor medida las competencias éticas. En cuanto al uso de las TIC, el personal es el que presenta mayor intensidad de la relación con las competencias tecnológicas, mientras que el académico no escolar con las competencias pedagógicas y tecnológicas. El uso académico en el aula es el que muestra menor intensidad de la relación con los tres componentes competenciales. La intensidad de las relaciones oscila en un rango que va desde .123 a .607. El tamaño del efecto es grande entre las competencias tecnológicas con el uso personal y con el uso académico no escolar; mediano entre las competencias tecnológicas y el uso académico en el aula y en todos los casos de relación de las competencias pedagógicas con los tres usos; y pequeño en el caso de la relación de las competencias éticas con los tres usos de las TIC.

Considerando el enfoque profundo, su relación con las dimensiones de las competencias TIC y las de uso de las TIC en todos los casos son positivas. Por ello, a mayor puntuación en el enfoque profundo mayor puntuación en las competencias TIC y viceversa, y mayor puntuación en los usos de las TIC y viceversa. La intensidad de las relaciones oscila entre .144 y .345. El tamaño del efecto es mediano en la relación competencias pedagógicas y enfoque profundo, mientras que en las restantes el tamaño del efecto es pequeño.

Finalmente, el enfoque superficial se relaciona negativamente con las competencias pedagógicas y las éticas, con un tamaño del efecto pequeño. Por lo tanto, a mayor puntuación en el enfoque superficial menor puntuación en las competencias pedagógicas y éticas, y viceversa. También se ha de destacar la relación positiva con el uso personal, si bien con un tamaño del efecto pequeño. Las restantes vinculaciones son insignificantes en su intensidad. 
Tabla 2

Correlación de Pearson entre las dimensiones de enfoques de aprendizaje, competencias TIC y uso TIC

\begin{tabular}{|c|c|c|c|c|c|c|c|c|c|}
\hline & & 1 & 2 & 3 & 4 & 5 & 6 & 7 & 8 \\
\hline 1. Enfoque superficial & & 1 & & & & & & & \\
\hline \multirow{2}{*}{ 2. Enfoque profundo } & $\mathrm{r}$ & $-.153^{* *}$ & 1 & & & & & & \\
\hline & $r^{2}$ & .023 & & & & & & & \\
\hline \multirow{2}{*}{ 3. Competencia tecnológica } & $\mathrm{r}$ & .068 & $.191^{* *}$ & 1 & & & & & \\
\hline & $r^{2}$ & .005 & .036 & & & & & & \\
\hline \multirow{2}{*}{ 4. Competencia pedagógica } & $\mathrm{r}$ & $-.150^{* *}$ & $.345^{* *}$ & $.487^{* *}$ & 1 & & & & \\
\hline & $r^{2}$ & .023 & .119 & .237 & & & & & \\
\hline \multirow{2}{*}{ 5. Competencia ética } & $\mathrm{r}$ & $-.182^{* *}$ & $.199^{* *}$ & $.198^{* *}$ & $.409^{* *}$ & 1 & & & \\
\hline & $\mathrm{r}^{2}$ & .033 & .040 & .039 & .167 & & & & \\
\hline \multirow{2}{*}{ 6. Uso personal } & $\mathrm{r}$ & $.129^{* *}$ & $.205^{* *}$ & $.607^{* *}$ & $.445^{* *}$ & $.173^{* *}$ & 1 & & \\
\hline & $r^{2}$ & .017 & .042 & .368 & .198 & .030 & & & \\
\hline \multirow{2}{*}{ 7. Uso académico no escolar } & $\mathrm{r}$ & .073 & $.265^{* *}$ & $.504^{* *}$ & $.473^{* *}$ & $.178^{* *}$ & $.759^{* *}$ & 1 & \\
\hline & $\mathrm{r}^{2}$ & .005 & .070 & .254 & .224 & .032 & .576 & & \\
\hline \multirow{2}{*}{ 8. Uso académico en el aula } & $\mathrm{r}$ & $.093^{*}$ & $.144^{* *}$ & $.407^{* *}$ & $.360^{* *}$ & $.123^{* *}$ & $.653^{* *}$ & $.791^{* *}$ & 1 \\
\hline & $\mathrm{r}^{2}$ & .009 & .021 & .166 & .130 & .015 & .426 & .626 & \\
\hline
\end{tabular}

** La correlación es significativa en el nivel .01.

* La correlación es significativa en el nivel .05.

\section{Relación entre las competencias TIC, el uso de las TIC y los enfoques de aprendizaje}

En este apartado se pretende conocer la relación entre el conjunto formado por las dimensiones de las competencias TIC -competencias tecnológicas, competencias pedagógicas y competencias éticas-, los tres usos de las TIC - uso personal, uso académico no escolar y uso académico en el aula- y los enfoques de aprendizaje -enfoque profundo y enfoque superficial-. Para ello, se ha realizado un análisis de correlación canónica no lineal, pues permite abordar las relaciones entre los tres conjuntos dimensionales.

A partir del ajuste-ver tabla 3- se ha optado por dos dimensiones, pues es la solución más parsimoniosa y consistente que explica adecuadamente la relación entre los tres conjuntos.

En este caso, como se puede ver en la tabla 4, la primera función explica un $14.48 \%$ (tamaño de efecto mediano) de la varianza compartida y la segunda función un 3.61\% (tamaño de efecto pequeño). Consiguientemente, la primera función es la que determina prácticamente la relación entre los tres conjuntos de dimensiones, siendo la segunda función una matización de la vinculación entre los tres conjuntos. 
Tabla 3

Resumen de análisis de la Correlación canónica no lineal

\begin{tabular}{|c|c|c|c|c|}
\hline & & \multicolumn{2}{|c|}{ Dimensión } & \multirow{2}{*}{ Suma } \\
\hline & & 1 & 2 & \\
\hline \multirow[t]{4}{*}{ Pérdida } & $\begin{array}{l}\text { Enfoques de } \\
\text { aprendizaje }\end{array}$ & .580 & .429 & 1.008 \\
\hline & Competencias TIC & .367 & .637 & 1.004 \\
\hline & Uso TIC & .292 & .554 & .846 \\
\hline & Media & .413 & .540 & .953 \\
\hline Autovalor & & .587 & .460 & \\
\hline Ajuste & & & & 1.047 \\
\hline
\end{tabular}

A partir de los coeficientes estructura, y siguiendo el criterio de Fan y Konold (2010) de considerar significativos los valores iguales o mayores de .32, en la primera función canónica -ver tabla 4- casi todas las dimensiones contribuyen a la explicación de la función, con excepción del enfoque superficial y la competencia ética. Tanto la competencia tecnológica como el uso personal de las TIC contribuyen más en la función, y en menor medida el uso académico en el aula. Respecto a las competencias TIC las competencias tecnológicas son las que aportan una mayor relevancia en esta primera función, con menor aportación por parte de las competencias pedagógicas. En cuanto al uso de las TIC, es el personal el más relevante, seguido por el uso académico no escolar y por último el uso académico en el aula. Del conjunto de enfoques de aprendizaje, el enfoque profundo es el que contribuye. Además, el signo de todas las dimensiones consideradas representa que mayores valores de las competencias tecnológicas y pedagógicas, mayor valor en el uso de las TIC y mayor valor en el enfoque profundo.

En la segunda función -ver tabla 4- los coeficientes estructura reflejan la relación del enfoque superficial con las competencias pedagógicas y éticas, y con el uso académico en el aula. El signo de la relación señala que el enfoque superficial se vincula negativamente con las competencias pedagógicas y las competencias éticas, con lo que a valores mayores del enfoque superficial menores valores en las competencias pedagógicas y éticas. En cambio, el enfoque superficial se relaciona positivamente con el uso académico en el aula, de modo que a mayor valor del enfoque superficial mayor uso académico en el aula de las TIC.

Finalmente, a partir de la figura 1, si se consideran de forma global ambas funciones se pueden establecer tres agrupaciones de las dimensiones. En la primera el enfoque superficial se asocia con el uso académico en el aula. En la segunda, se produce la vinculación de las competencias tecnológicas con el uso personal, ocupando un papel central entre las otras dos agrupaciones. En la tercera, se produce la asociación del uso académico no escolar, el enfoque profundo y las competencias pedagógicas y las éticas, destacando que se opone a la primera agrupación -enfoque superficial y uso académico en el aula-. 


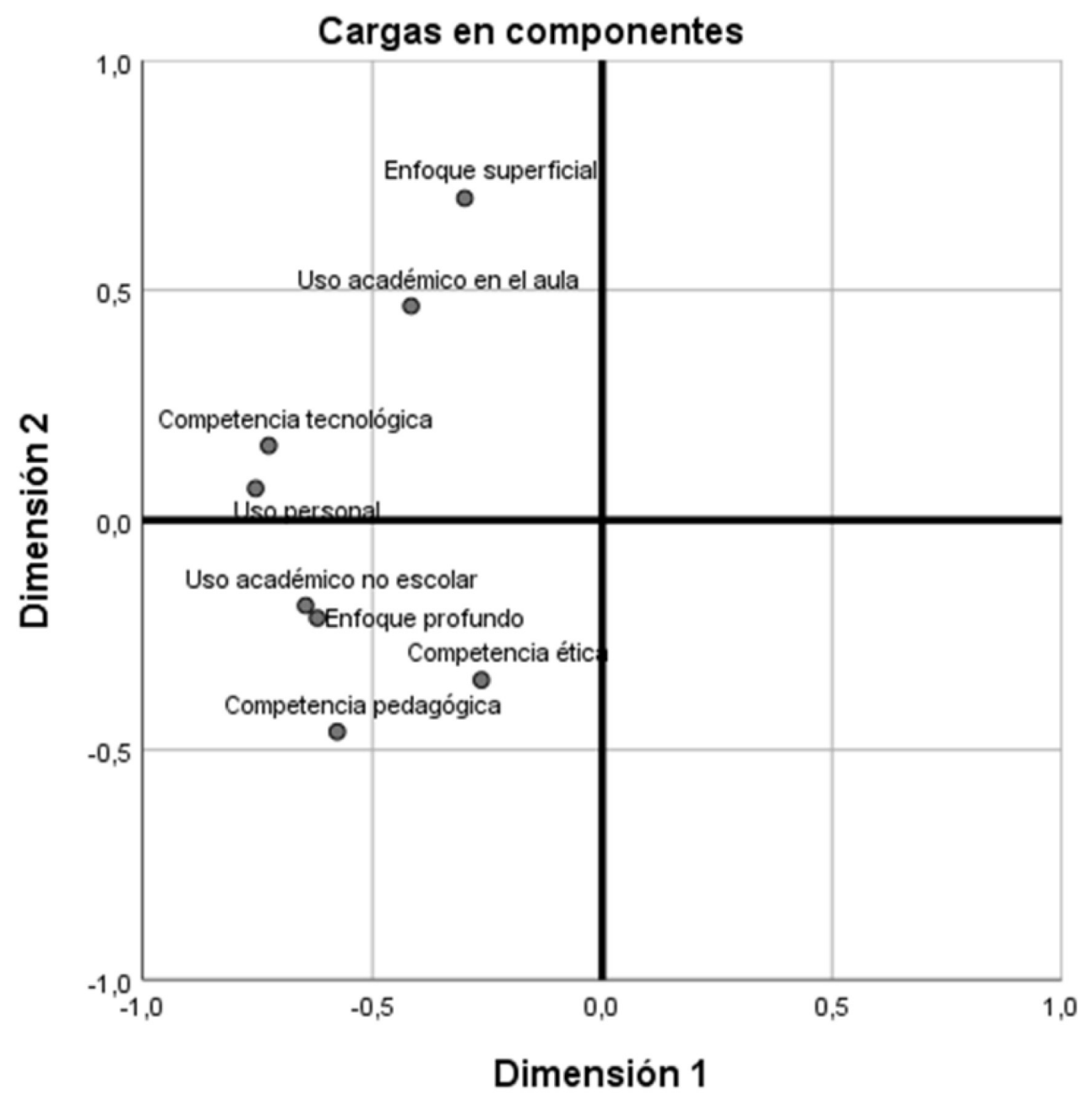

Figura 1. Diagrama bidimensional de las dos primeras dimensiones del análisis canónico. 


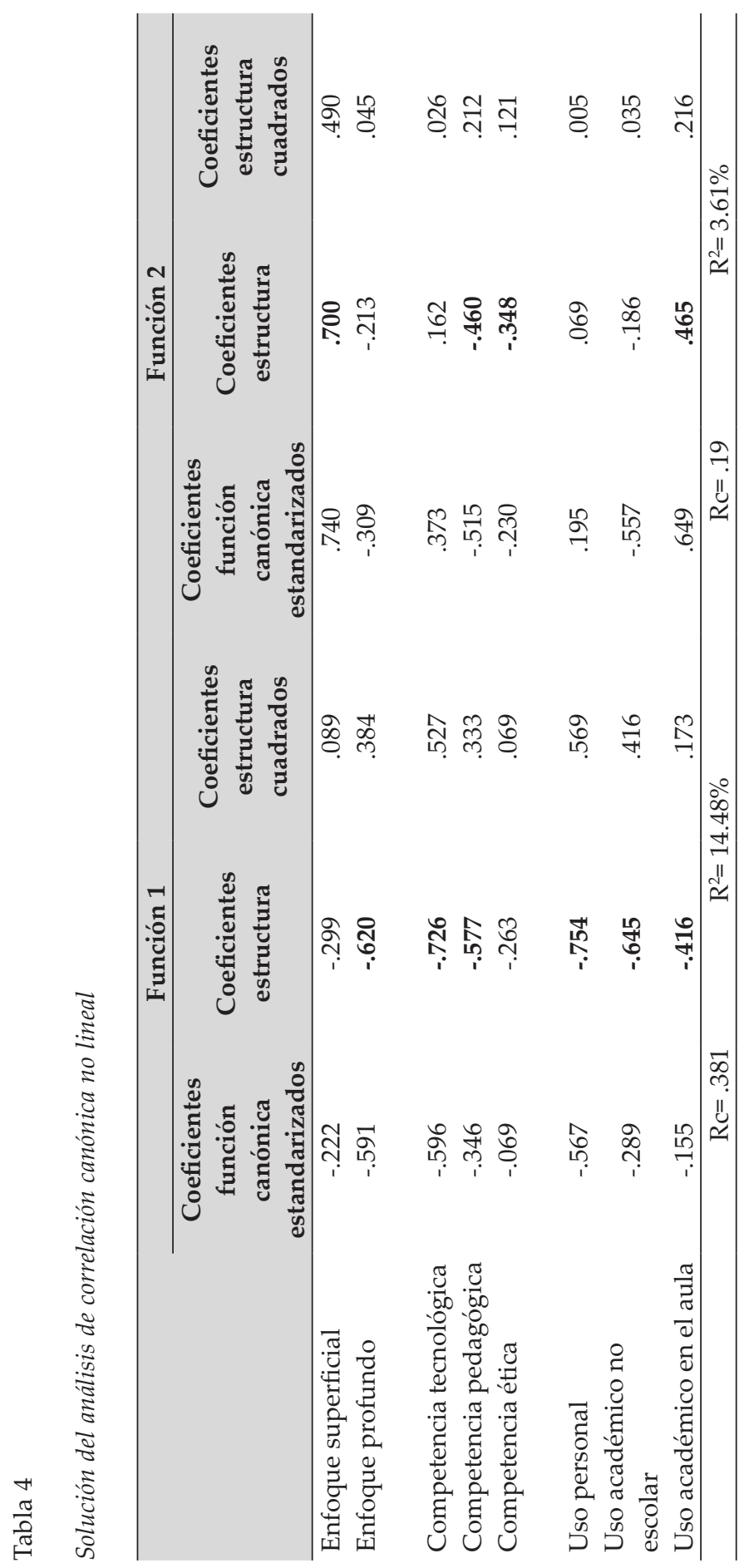




\section{Discusión y conclusiones}

En primer lugar, los resultados obtenidos caracterizan el alumnado de los grados de Pedagogía y Educación Social de la Universidad de Valencia por un enfoque de aprendizaje profundo, coincidente con la investigación de Mirete Ruiz, Pérez Rubio y Maquilón (2018); con un uso de las TIC básicamente personal y académico no escolar y para ciertas tareas de manera regular, coincidente con Fraillon, Ainley, Schulz, Friedman y Gebhardt (2014); y con un nivel de domino medio en las competencias TIC, mayor en las competencias éticas y menor en las tecnológicas, coincidente con otros estudios (Arras et al., 2011; Almerich et al., 2020).

En segundo lugar, a partir de la relación univariada se ha encontrado que los tres subconjuntos competenciales en TIC se vinculan positivamente con los tres usos de las TIC, coincidente con los resultados de Rohatgi et al. (2016). Así, un incremento de las competencias TIC se vincula con un incremento del uso de las TIC. Se ha de destacar que las competencias tecnológicas presentan mayor intensidad en su vinculación con el uso de las TIC que los otros dos componentes competenciales. Asimismo, tanto el uso personal y el académico no escolar muestran mayor relación con las competencias TIC, vinculándose predominantemente el primero a las competencias tecnológicas y el segundo a las competencias pedagógicas y éticas. El uso académico de las TIC en el aula se relaciona, aunque con menor intensidad que los otros dos usos, de forma positiva con los tres subconjuntos competenciales, a diferencia de la investigación de Hatlevik et al. (2015) que encontraron una relación negativa entre las competencias TIC y el uso en la escuela.

El enfoque de aprendizaje profundo se vincula de forma positiva tanto con las competencias TIC como con el uso de las TIC, de modo que un incremento del enfoque profundo supone un incremento en las competencias y uso de los recursos tecnológicos. Estos resultados coinciden con el estudio de Maquilón Sánchez et al. (2013) en cuanto al uso de las TIC, y la conexión del enfoque profundo con el uso académico no escolar encontrado por Díaz-García et al. (2019). No obstante, el tamaño del efecto es pequeño en casi todas las relaciones, a excepción de la relación con las competencias pedagógicas que es mediano. En cuanto al enfoque de aprendizaje superficial se vincula de forma negativa con las competencias pedagógicas y éticas, y de forma positiva con el uso personal de las TIC, con un tamaño del efecto pequeño.

La relación entre el enfoque de aprendizaje profundo y superficial es negativa, coincidente con el estudio de Mirete Ruiz et al. (2018), con un tamaño del efecto pequeño.

En tercer lugar, en la relación multivariada de los tres conjuntos considerados se ha encontrado, a partir de la primera función, que existe una primera vinculación entre las competencias tecnológicas y pedagógicas, los tres tipos de uso de las TIC y el enfoque profundo. El signo de la función indica una relación positiva entre las anteriores dimensiones, de modo que un incremento en el enfoque de aprendizaje profundo representa un incremento en las competencias tecnológicas y pedagógicas y en los tres tipos de uso de las TIC, con un tamaño del efecto mediano.

En cuanto a la segunda función, se ha de destacar la relación positiva del enfoque de aprendizaje superficial con el uso académico de las TIC en el aula, y negativa con las competencias pedagógicas y éticas, con un tamaño del efecto pequeño. Así, un 
incremento en el enfoque de aprendizaje superficial se vincula con un incremento en el uso de las TIC en el aula, y una disminución en las competencias pedagógicas y éticas.

Para concluir, respecto al primer objetivo se ha encontrado una evidencia a nivel multivariado de la relación compleja entre las competencias TIC, el uso de las TIC y los enfoques de aprendizaje, diferente a la aportada por la perspectiva univariada. Consecuentemente, es necesario abordar estudios multivariados para determinar relaciones complejas, como son las TIC en educación (Almerich et al., 2020; Hatlevik et al., 2015), que la perspectiva univariada no logra advertir.

En cuanto al segundo objetivo, las dimensiones determinantes son el enfoque profundo del aprendizaje, las competencias tecnológicas y pedagógicas y los tres usos de las TIC, estableciéndose que el enfoque de aprendizaje profundo se vincula positivamente con las dos dimensiones competenciales y los tres usos de las TIC. En menor medida, tanto el enfoque de aprendizaje superficial como las competencias éticas son menos determinantes en la relación.

De forma global, a partir del análisis canónico no lineal se ha establecido una primera agrupación de dimensiones constituida por las competencias pedagógicas y las éticas, el uso académico no escolar de las TIC y el enfoque de aprendizaje profundo. La importancia de este vínculo se refleja por la relación de las competencias pedagógicas, en las que se sitúa la búsqueda y gestión de la información - análisis, evaluación y síntesis- mediante los recursos tecnológicos (Almerich et al., 2020), con el enfoque profundo que supone la relación entre ideas y el establecimiento de conexiones con el propio conocimiento. En cierto modo, este vínculo queda avalado por el estudio de Richardson et al. (2012) que señala la relación positiva del enfoque profundo con el rendimiento académico y por el estudio de Alderete, Di Meglio y Formichella (2017) en el que encontraron que el uso académico no escolar de las TIC incrementa el rendimiento académico.

Una segunda agrupación es la fuerte conexión positiva entre las competencias tecnológicas y el uso personal de las TIC. La posición intermedia de esta agrupación representa el papel básico de ambas dimensiones, tanto en la adquisición de competencias pedagógicas (Almerich et al., 2020) como en el uso académico no escolar.

La tercera agrupación es entre el uso académico en el aula y el enfoque de aprendizaje superficial. Esto representa que el alumnado no realiza en el aula un uso adecuado de las TIC, pues no se favorece la relación entre ideas, sino más bien la sustitución de los recursos tradicionales del proceso de enseñanza-aprendizaje (libro, apuntes, etc.) por los recursos tecnológicos. Este hallazgo se ve reforzado por la vinculación negativa del uso académico en el aula con el rendimiento como demostraron Alderete et al. (2017).

Este estudio presenta limitaciones, pues la muestra es del ámbito de la educación de una universidad y seleccionada mediante un muestreo no probabilístico. Por ello, es fundamental que otros estudios corroboren o refuten los hallazgos encontrados, mediante muestras más amplias y con titulaciones de otros ámbitos. Además, se ha utilizado cuestionarios de autopercepción de las competencias TIC, siendo necesario considerar en otros estudios medidas directas de las mismas.

Para finalizar, el uso de las TIC por parte del alumnado de educación se constituye en una dimensión fundamental para la adquisición de las competencias TIC y el enfoque de aprendizaje, además de convertirse en una estrategia de elemento facilitador del aprendizaje (García-Valcárcel \& Tejedor, 2017). Sin embargo, se debe potenciar un 
mayor y mejor uso de las TIC en el aula, dado que el aprendizaje más significativo se produce cuando el alumnado usa las TIC para realizar tareas académicas en casa, pues el uso de las TIC en el aula presencial conlleva un aprendizaje más superficial (Díaz-García et al., 2019). Es necesario, por tanto, que se realice un esfuerzo por crear entornos de aprendizaje que favorezcan las competencias pedagógicas y éticas y el aprendizaje profundo cuando el alumnado usa las TIC en el aula.

\section{Referencias}

017/07/ACTADIPE_2017.pdf

849-856. https://aidipe2017.aidipe.org/files/2017/07/ACTAS_AIDIPE_2017.pdf

Actas XVIII Congreso Internacional de Investigación Educativa. Interdisciplinariedad $\mathrm{y}$ transferencia. pp

Actas XVIII Congreso Internacional de Investigación Educativa. Interdisciplinariedad $\mathrm{y}$ transferencia. pp

Almerich, G.;Orellana, N.;Bo-Bonet, R.;Checa, S. (2017). Relación entre la búsqueda y gestión de la

Almerich, G.;Orellana, N.;Bo-Bonet, R.;Checa, S. (2017). Relación entre la búsqueda y gestión de la

849-856. https://aidipe2017.aidipe.org/files/2

Alderete, M. V., Di Meglio, G., \& Formichella, M. M. (2017). Acceso a las TIC y rendimiento educativo: ¿una relación potenciada por su uso? Un análisis para España. Revista de Educación, 377, 54-81. doi: 10.4438/1988-592X-RE-2017-377-353

Almerich, G., Suárez-Rodríguez, J., Díaz-García, I., \& Orellana, N. (2020). Estructura de las competencias del siglo XXI en alumnado del ámbito educativo. Factores personales influyentes. Educación XX1, 23(1), 45-74, doi: 10.5944/educXX1.23853

Arras, A. M. D. G., Torres, C. A., \& García-Valcárcel, A. (2011). Competencias en Tecnologías de Información y Comunicación (TIC) de los estudiantes universitarios. Revista latina de comunicación social, (66), 130-152. Recuperado de http://www.revistalatinacs.org/11/art/927_Mexico/06_Arras.html.

Biggs, J., Kember, D., \& Leung, D. Y. (2001). The revised two-factor study process questionnaire: R-SPQ-2F. British journal of educational psychology, 71(1), 133-149. doi: 10.1348/000709901158433

Biggs, J., \& Tang, C. (2011). Teaching For Quality Learning At University. London: McGraw-Hill Education. Recuperado de https://pdfs.semanticscholar.org/1f96/ f4a66767293cf2902652ac9eadfa8ef51f13.pdf

Comisión Europea (2017). Comunicación de la Comisión al Parlamento Europeo, al Consejo, al Comité Económico y Social Europeo y al Comité de las Regiones sobre una agenda renovada de la UE para la educación superior. Recuperado de https://eur-lex.europa. eu/legal-content/ES/TXT/PDF/?uri=CELEX:52017DC0247\&from=es.

Comisión Europea (2018). ANEXO de la Propuesta de Recomendación del Consejo relativa a las competencias clave para el aprendizaje permanente. Recuperado de https:/eur-lex. europa.eu/resource.html?uri=cellar:395443f6fb6d11e7b8f501aa75ed71a1.0013.02/ DOC2\&format=PDF. 
Cohen, J., Cohen, P., West, S. G., \& Aiken, L. S. (2003). Applied multiple regression/correlation analysis for the behavioral sciences. London: Routledge.

Díaz-García, I., Almerich, G., Cebrián-Cifuentes, S., \& Suárez-Rodríguez, J. (2019). La Influencia del Uso de las TIC sobre los Enfoques de Aprendizaje en Alumnado Universitario del Ámbito de la Educación. En J. Murillo \& C. Martínez-Garrido (Coords.), Actas del XIX Congreso Internacional de Investigación Educativa. Metodología de investigación educativa (Volumen II, pp. 301-306). Madrid: AIDIPE.

Fan, X., \& Konold, T. R. (2010). Canonical correlation analysis. En G. R. Hancock y R. O. Mueller (Eds), The reviewer's guide to quantitative methods in the social sciences (pp. 29-40). New York: Routledge.

Fraillon, J., Ainley, J., Schulz, W., Friedman, T., \& Gebhardt, E. (2014). Preparing for life in a digital age: The IEA international computer and information literacy study international report. Cham: Springer.

García-Valcárcel, A., \& Tejedor, F. (2017). Percepción de los estudiantes sobre el valor de las TIC en sus estrategias de aprendizaje y su relación con el rendimiento. Educación XX1, 20(2), 137-159. doi: 10.5944/educXX1.19035

Gargallo, B., Garfella, P. R., \& Pérez, C. (2006). Enfoques de aprendizaje y rendimiento académico en estudiantes universitarios. Bordón, 58(3), 45-61. Recuperado de https:// recyt.fecyt.es/index.php/BORDON/article/view/39598

Hatlevik, O. E., Ottestad, G., \& Throndsen, I. (2015). Predictors of digital competence in 7th grade: a multilevel analysis. Journal of Computer Assisted Learning, 31(3), 220231. doi: $10.1111 /$ jcal.12065

ISTE (2016). ISTE Standards for Students. Recuperado de https://www.iste.org/standards/ for-students.

Maquilón Sánchez, J. J., Mirete Ruiz, A. B., García Sánchez, F. A., \& Hernández Pina, F. (2013). Valoración de las TIC por los estudiantes universitarios y su relación con los enfoques de aprendizaje. Revista de Investigación Educativa, 31(2), 537-554. doi: 10.6018/rie.31.2.151891

Martínez-Clares, P., \& González-Lorente, C. (2019). Competencias personales y participativas vinculantes a la inserción laboral de los universitarios: Validación de una escala. RELIEVE, 25(1). Recuperado de https://www.uv.es/RELIEVE/v25n1/ RELIEVEv25n1_6.pdf.

Marton, F., \& Säljö, R. (1976a) On qualitative differences in learning: I. Outcome and process. Journal of Educational Psychology, 46(1), 4-11. doi: 10.1111/j.2044-8279.1976. tb02980.x

Marton, F., \& Säljö, R. (1976b) On qualitative differences in learning: II. Outcome as a function of the learner's conception of the task. Journal of Educational Psychology, 46(2), 115-127. doi: 10.1111/j.2044-8279.1976.tb02304.x

McCune, V., \& Entwistle, N. (2011). Cultivating the disposition to understand in 21st century university education. Learning and Individual Differences, 21(3), 303-310. doi: 10.1016/j.lindif.2010.11.017

Mirete Ruiz, A. B., Pérez Rubio, R., \& Maquilón, J. J. (2018). Estudio comparativo de los enfoques de aprendizaje de los estudiantes de los Grados en Pedagogía y Educación Primaria. Educatio Siglo XXI, 36(1), 173-194. doi:10.6018/j/324221 
Monroy, F., \& Hernández Pina, F. (2014). Factores que influyen en los enfoques de aprendizaje universitario. Una revisión sistemática. Educación XX1, 17(2), 105-124. doi: 10.5944/educxx1.17.2.11481

Nugent A., Lodge, J. M., Carroll, A., Bagraith, R., MacMahon, S., Matthews, K. E., \& Sah, P. (2019). Higher Education Learning Framework: An evidence informed model for university learning. Brisbane: The University of Queensland. Doi: 10.13140/RG.2.2.20761.47207.

REBIUN (2016). Marco de competencia digital para estudiantes de grado. CRUE- REBIUN. Recuperado de http://rebiun.xercode.es/xmlui/bitstream/handle/20.500.11967/65/ IIIPE_Linea2_2016_Marco_de_competencia_digital_para_estudiantes_de_grado_ adaptacion_DIGCOMP_.pdf? sequence $=4 \&$ isAllowed $=$. .

Richardson, M., Abraham, C., \& Bond, R. (2012). Psychological correlates of university students' academic performance: A systematic review and meta-analysis. Psychological Bulletin, 138(2), 353-387. doi: 10.1037/a0026838

Rohatgi, A., Scherer, R., \& Hatlevik, O. E. (2016). The role of ICT self-efficacy for students' ICT use and their achievement in a computer and information literacy test. Computers \& Education, 102, 103-116. doi:10.1016/j.compedu.2016.08.001

Soler-Contreras, M. G., Cárdenas-Salgado, F. A., Hernández-Pina, F., \& MonroyHernández, F. (2017). Enfoques de aprendizaje y enfoques de enseñanza: origen y evolución. Educación y Educadores, 20(1), 65-88. doi: 10.5294/edu.2017.20.1.4

Suárez-Rodríguez, J.M., Almerich, G., Orellana, N., \& Díaz-García, I. (2016). Protocolo Innovatic. Documento no publicado.

Vuorikari, R., Punie, Y., Carretero Gomez S., y Van den Brande, G. (2016). DigComp 2.0: The Digital Competence Framework for Citizens. Update Phase 1: The Conceptual Reference Model. Sevilla: Unión Europea.

Fecha de recepción: 9 de enero de 2020.

Fecha de revisión: 17 de febrero de 2020.

Fecha de aceptación: 8 de abril de 2020 . 